\title{
Cognición de la Innovación Industrial en América Latina: Avances y Desafíos
}

\author{
Cognition of Industrial Innovation in Latin America: \\ Advances and Challenges
}

\author{
Fernando Salazar', Judith Cavazos², Jordi Poch ${ }^{3}$, Felipe Santos ${ }^{4}$
}

\begin{abstract}
El presente artículo, hace un estudio comparativo y relacional de los niveles y avances tecnológicos que llevan a la sociedad a un uso más eficaz de los recursos productivos y a transformar ideas nuevas en soluciones viables a través de productos y servicios, paradigmas y procesos que con el desarrollo de la nueva revolución tecnológica, la innovación entendida en este trabajo como una nueva idea o enfoque que se aplica en nuevas formas de crear valor para la organización y otras partes interesadas en el bien de la humanidad. El valor de la innovación requiere que las organizaciones desarrollen capacidades tecnológicas internas y capacidad de conocimiento. Este artículo describe el proceso de cómo es que no siempre se pueden ver los resultados de trabajos empíricos y que son consistentes, de hecho la falta de significancia de la innovación hacia la productividad no es exclusiva de las economías de América Latina. Se plantea la discusión de cómo puede deberse a diferentes circunstancias que rodean a la innovación y sus efectos en el largo plazo, como el hecho de que las empresas están muy lejos de la frontera tecnológica y por los débiles o inexistentes incentivos para invertir en innovación.
\end{abstract}

Keywords: innovación industrial, investigación y desarrollo, américa latina, índice de innovación, cognición.

This paper makes a comparative study and relational levels and technological advances that lead society to a more efficient use of productive resources and transform new ideas into viable solutions through products and services, paradigms and processes with development of the new technological revolution, innovation understood in this work as a new idea or approach applied in new ways to create value for the organization and other stakeholders in the good of humanity. The value of innovation requires organizations to develop internal technological capabilities and knowledge capacity. This article describes the process of how you can't always see the results of empirical studies are consistent and in fact the lack of significance of innovation to productivity is not exclusive to Latin American economies. Discussion of how it can be due to different circumstances surrounding innovation and its effects in the long run, the fact that companies are far from the technological frontier and weak or no incentives to invest in innovation arises.

Keywords: industrial innovation, research and development, latin america, innovation index, cognition.

\footnotetext{
1,4Escuela de Administración, Universidad del Rosario, Calle I2C \#6-25, Bogotá, Colombia, 2970200.e-mail:andres.santos@urosario.edu.co ${ }^{2}$ Centro Interdisciplinario de Posgrados e Investigación, Universidad Popular Autónoma del Estado de Puebla-UPAEP,

Calle 2 I sur \#I I03, Puebla, México, C.P., 724I0, 2299400. e-mail: judith.cavazos@upaep.mx

3Departament de Informàtica, Matemàtica Aplicada i Estadística, Universitat de Girona, Plaça Sant Domènec 3, I707I, Girona, Catalunya, España. Phone: 9724I84I3.e-mail: poch@imae.udg.edu
}

ISSN: 07 I8-2724. (http://www.jotmi.org)

Journal of Technology Management \& Innovation (c) Universidad Alberto Hurtado, Facultad de Economía y Negocios. 


\section{Introducción}

Con el desarrollo de la nueva revolución tecnológica, la innovación, entendida en este trabajo como una nueva idea o enfoque que se aplica en nuevas formas de crear valor para la organización y otras partes interesadas, sean los clientes, proveedores, asociados, comunidades, gobiernos, o incluso en el bien de la humanidad (Lee, Olson y Trimi, 20I I0) (Katragada, 2006), se ha convertido en una de las principales fuerzas para impulsar el desarrollo industrial. Así, la investigación sobre este tema está desempeñando un papel crítico para la organización industrial, a través de la innovación vertical y horizontal. La innovación vertical también conocida como innovación industrial, incrementa la productividad de nuevos bienes intermedios en la cadena de la industria, generando una escalera de la calidad que conducirá a una versión de mayor calidad del bien intermedio, terminando por expulsar del mercado a la versión existente hasta entonces, tratándose principalmente de lo que Shumpeter denominó como una destrucción creativa. Por otro lado, está la innovación horizontal que creará mayor variedad de bienes intermedios, y por lo tanto mayor especialización y eficiencia, aunque no mayor progreso técnico, permitiendo producir más bienes finales que conviven con los productos antiguos (Borondo, 2008); (Li y Wang, 20I0).

La innovación industrial es una de las bases de las ventajas competitivas sostenibles de las organizaciones. Se ha aprendido a lo largo de décadas que el gasto en Investigación y Desarrollo (I+D), innovación, productividad e ingreso per cápita influyen en el crecimiento sostenido a largo plazo (Rouvinen, 2002). Sin embargo, en los países emergentes la imitación y la adquisición de tecnologías son más importantes que la I+D y la innovación como condiciones previas para aprender y ponerse al día (Bell y Pavitt, 1993). Por ello, el desafío de los acuerdos industriales bilaterales entre países es que los innovadores con menos conocimiento tecnológico logren una convergencia a un país con más conocimiento tecnológico (Grupp, 1998).

La I + D extranjera tiene más efecto en un país cuanto más abierto al comercio, cuanta más I + D se hace en el propio país y cuanto mayor es nivel educativo, porque el país es entonces capaz de asimilar mejor la tecnología extranjera (Borondo, 2008), esto es lo que conoce como "Capacidad de Absorción" es decir, la habilidad de una organización o este caso de un país para reconocer aquellos conocimientos nuevos y valiosos, asimilarlos con el conocimiento existente y aplicarlos en fines comerciales y/o en la creación de nuevas capacidades (Cohen y Levinthal, 1990); (Collins y Hitt, 2006); (Zhao y Anand, 2009). Mirando hacia el futuro, en Europa la "Comisión de Investigación y Desarrollo hacia el 2020" ha considerado tres pilares de desarrollo: I) Competitividad industrial, retos sociales y excelencia con el fin de crear mayor valor agregado en Europa, 2) La creación de un factor competitivo vía la eficiencia y la sostenibilidad (Re-uso y reciclaje de equipo de manufactura y de productos) y, 3) Una manufactura con enfoque humano y personalización para una competencia global y local. Así, en este artículo analizamos la innovación industrial en América Latina, reflexionando sobre sus avances y los desafíos que enfrenta en las primeras décadas del siglo XXI.

\section{Método \\ La Realidad Latinoamericana}

El valor de la innovación requiere que las organizaciones desarrollen capacidades tecnológicas internas y capacidad de conocimiento. En la región latinoamericana las organizaciones principalmente se han centrado en obtener la fuente de innovación exclusivamente de las inversiones en I+D, por lo que los resultados sobre innovación y productividad han sido mixtos (Crespi y Zuniga, 2012). Sin embargo, es necesario que se consideren los esfuerzos de innovación más allá de la I+D. Crespi y Zuniga (20I2) estudiaron seis países latinoamericanos y encontraron que existe una debilidad de las empresas para vincularse con el sistema de innovación nacional y para integrar los recursos científicos y tecnológicos en las estrategias de innovación.

El Índice de Innovación Global (GII) es un indicador compuesto que clasifica a 142 países en función de que tan favorable es su entorno hacia la innovación y sus resultados, basándose en "entrada" y "salida" de la innovación. Los pilares de entrada implican: instituciones, capital humano e investigación, infraestructura, sofisticación del mercado y sofisticación de negocios. Los pilares de salida incluyen resultados en conocimiento y tecnología y salidas creativas. Cada uno de los pilares implican sub-pilares y éstos se componen de indicadores individuales, sumando 84 en total (Global Innovation Index, 20I0).

Los últimos años, Suiza ha encabezado la lista y países africanos como Argelia y Sudán se reportan en los últimos lugares (Global Innovation Index, 2010). En el caso de Latinoamérica los reportes de 2009 a 2013 evidencian desafíos importantes para la región (ver tabla I y gráfica I). Chile y Costa Rica han presentado los mejores resultados, pero en general a la región se le describe formada por economías de medianos ingresos o ingresos por arriba de la media, cuyos resultados en el ranking de innovación muestran grandes disparidades. Cuando se analizan los subíndices, Chile evidencia fortalezas en todos, excepto en Capital Humano e Investigación, evidenciándose deficiencias en el sector educativo terciario, instituciones y resultados creativos (GII, 20II). Por otro lado, la mejora de la posición de Costa Rica se ha debido principalmente a su desempeño en los subíndices "resultados", especialmente en eficiencia, 
conocimiento (absorción y difusión) y tecnología. El país logró mejores vínculos con mercados extranjeros de conocimiento. Además, también ha mejorado en instituciones, y la sofisticación del mercado y las empresas (GII, 20I2). No obstante, aún hay muchos desafíos para el desarrollo de su potencial innovador especialmente en la mejora de las condiciones de crédito y la inversión (GII, 20I0), en el desarrollo de su capital humano, la investigación y la mejora de su infraestructura (GII, 20I3).

Recientemente varios países y ciudades de la región han establecido nuevos instrumentos a fin de mejorar los ecosistemas locales (GII, 20I3), esperando que sus esfuerzos reflejen resultados en los siguientes años. Particularmente, el caso de Brasil es de interés, porque realizó varios ajustes para orientarse hacia la innovación. Su mejor desempeño ha sido en el subíndice de "resultados", cuenta con infraestructura, sofisticación de negocios, desarrolla conocimiento y tecnología, tiene las tres universidades mejor posicionadas en la región, familias de patentes (GIl, 20I3) y un desempeño logístico aceptable posicionándose como cuarto en la región, lugar 65 según el Logistics Performance Index (LPI, 2014) después de Chile (lugar 42 en el mundo y primero en Latinoamérica). No obstante, Brasil presenta fuertes desafíos en eficiencia, ambiente de negocios, educación terciaria, condiciones de crédito y comercio (GII, 20I3), instituciones, sofisticación del mercado, capital humano e investigación y salidas creativas (GII, 20I3).
Resultados de la Organización para la Cooperación y Desarrollo Económico (OECD, 2009), evidencian que más del $55 \%$ de los graduados de doctorado en Chile, Francia y China provienen de campos de Ciencias e Ingenierías. Además, el organismo reporta a Chile, Irlanda y Dinamarca como los países que mayor contribución hacen en el sector de la educación superior hacia la investigación básica (OECD, 20 I la). Mientras que Brasil,Alemania e Israel han presentado la mayor proporción de innovaciones no tecnológicas, donde más del $85 \%$ de las grandes empresas y más de dos tercios de sus pymes introdujeron innovaciones de marketing entre 2006 y 2008, aunque Chile se reportó como uno de los países de la OCDE menos innovadores en esta materia (OECD, 20l lb). Muchas empresas que desarrollan estrategias de innovación, no siempre las sustentan en Investigación y Desarrollo ni en la colaboración. Entre los países de la OECD, en Chile y Corea, el estatus de la I+D no parece estar infiltrada la colaboración en la innovación como sucede por ejemplo, en Reino Unido. Más del $90 \%$ de los innovadores de productos no están dedicados a I+D en Chile y Brasil (OECD, 20 I Ic). Por otro lado, el Tratado de Cooperación en materia de Patentes [PCT] (WIPO, 20I3), reportó que Brasil concentró en 2012 poco más de la mitad de las aplicaciones de patentes $(53 \%)$ de la región latinoamericana, seguido por México (17.1\%) y Chile (10.6\%), (Ver tabla 2). La mayor parte de las aplicaciones provienen de empresas, seguido de aplicaciones individuales, instituciones educativas y finalmente de gobiernos.

\begin{tabular}{|c|c|c|c|c|c|c|}
\hline País & $\begin{array}{c}\text { Lugar } \\
2009\end{array}$ & $\begin{array}{c}\text { Lugar } \\
2010\end{array}$ & $\begin{array}{c}\text { Lugar } \\
2011\end{array}$ & $\begin{array}{c}\text { Lugar } \\
2012\end{array}$ & $\begin{array}{c}\text { Lugar } \\
2013\end{array}$ & $\begin{array}{c}\text { Diferencia } \\
2012-2013\end{array}$ \\
\hline Chile & 39 & 42 & 38 & 39 & 46 & -7 \\
\hline Brasil & 50 & 68 & 47 & 58 & 64 & -6 \\
\hline Costa Rica & 48 & 41 & 45 & 60 & 39 & 21 \\
\hline Colombia & 75 & 90 & 71 & 65 & 60 & 5 \\
\hline Uruguay & 80 & 53 & 64 & 67 & 52 & 15 \\
\hline Argentina & 84 & 75 & 58 & 70 & 56 & 14 \\
\hline Perú & 85 & 88 & 83 & 75 & 69 & 6 \\
\hline Guatemala & 81 & 95 & 86 & 99 & 87 & 12 \\
\hline El Salvador & 88 & 91 & 90 & 93 & 88 & 5 \\
\hline México & 61 & 69 & 81 & 79 & 63 & 16 \\
\hline Belice & ND & N/D & ND & 80 & 102 & -22 \\
\hline Paraguay & 118 & 127 & 74 & 84 & 100 & -16 \\
\hline Panamá & 67 & 66 & 77 & 87 & 86 & 1 \\
\hline Ecuador & 109 & 126 & 93 & 98 & 83 & 15 \\
\hline Bolivia & 123 & 129 & 112 & 114 & 95 & 19 \\
\hline Venezuela & 101 & 124 & 102 & 118 & 114 & 4 \\
\hline
\end{tabular}

Tabla I. Posición de países latinoamericanos en el Índice de Innovación Global 2009-20I3

ISSN: 07 I8-2724. (http://www.jotmi.org)

Journal of Technology Management \& Innovation (c) Universidad Alberto Hurtado, Facultad de Economía y Negocios. 
Existe una correlación positiva entre la innovación de producto y la productividad, pero suele ser más alta en organizaciones de tamaño grande y en el sector manufacturero que en el sector servicios, se sabe que las empresas que más invierten, desarrollan más productos, procesos o patentes. Los países emergentes que mejores resultados han mostrado entre la asociación I+D, innovación y productividad son
Corea del Sur, Malasia, Taiwán y China (OECD, 20IId). Sin embargo, en el caso de América latina también hay evidencias empíricas documentadas de esta relación (Arza y López, 2010); (Raffo, Lhuillery y Miotti, 2008). También (Crespi y Zuniga, 2012) corrieron un modelo considerando los datos de seis países de la región encontrando que la relación entre innovación y productividad es significativa. Sin em-

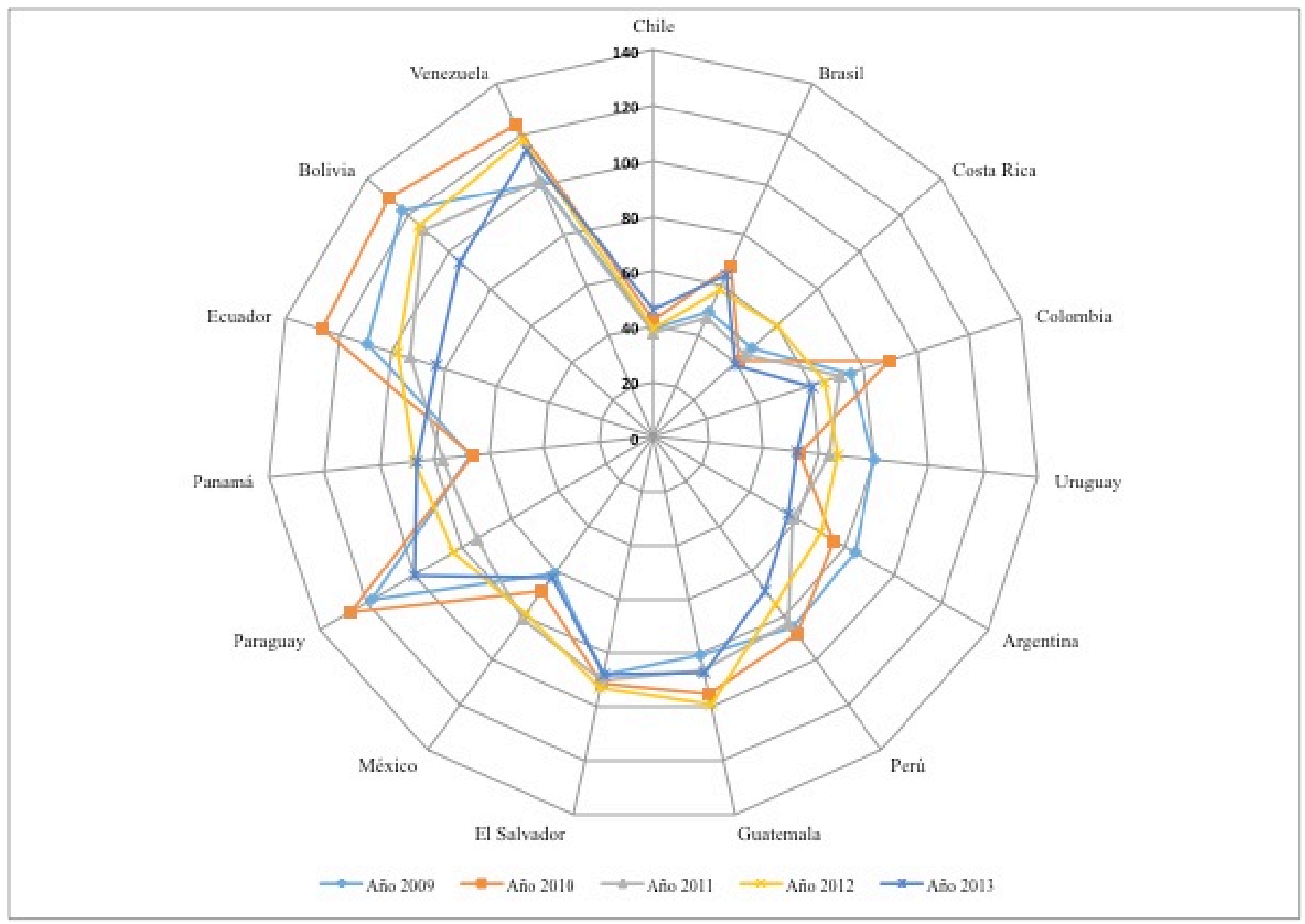

Gráfica I. Ranking de países latinoamericanos en el GII 2009-20I3

\begin{tabular}{|l|c|c|c|c|c|c|c|}
\hline País & \multicolumn{5}{|c|}{ Aplicaciones } & $\begin{array}{c}\text { Porcentaje Re- } \\
\text { gional }\end{array}$ & $\begin{array}{c}\text { Cambio com- } \\
\text { parado }\end{array}$ \\
\cline { 2 - 8 } & $\mathbf{2 0 0 8}$ & $\mathbf{2 0 0 9}$ & $\mathbf{2 0 1 0}$ & $\mathbf{2 0 1 1}$ & $\mathbf{2 0 1 2}$ & $\mathbf{2 0 1 2}(\mathbf{\%})$ & $\mathbf{2 0 1 1 - 2 0 1 2 ~ ( \% ) ~}$ \\
\hline Brasil & 472 & 492 & 488 & 564 & 587 & 53 & 4.1 \\
\hline México & 203 & 194 & 191 & 225 & 190 & 17.1 & -15.6 \\
\hline Chile & 27 & 54 & 88 & 118 & 118 & 10.6 & 0.0 \\
\hline Colombia & 37 & 63 & 46 & 57 & 73 & 6.6 & 28.1 \\
\hline Ecuador & 4 & 4 & 33 & 33 & 45 & 4.1 & 36.4 \\
\hline Otros & 99 & 75 & 54 & 88 & 95 & 8.6 & 8.0 \\
\hline Total & $\mathbf{8 4 2}$ & $\mathbf{8 8 2}$ & $\mathbf{9 0 0}$ & $\mathbf{1 , 0 8 5}$ & $\mathbf{1 , 1 0 8}$ & $\mathbf{1 0 0 . 0}$ & $\mathbf{2 . 1}$ \\
\hline
\end{tabular}

Tabla 2.Aplicaciones PCT en América Latina 20II-20I2 (WIPO, 20I3)

ISSN: 07 I8-2724. (http://www.jotmi.org) 
bargo, cabe mencionar que algunos estudios realizados en la región, han presentado resultados no compatibles con lo mencionado (Álvarez, Bravo y Navarro, 2010) e incluso no significativos para algunos países (Benavente, 2006); (Raffo, Lhuillery y Miotti, 2008).

Como puede verse no siempre los resultados de trabajos empíricos son consistentes, de hecho la falta de significancia de la innovación hacia la productividad no es exclusiva de las economías de América Latina. Esto puede deberse a diferentes circunstancias que rodean a la innovación y sus efectos en el largo plazo, como el hecho de que las empresas están muy lejos de la frontera tecnológica y por los débiles o inexistentes incentivos para invertir en innovación (Crespi y Zuniga, 20I2). Los países en desarrollo suelen presentar un ecosistema de innovación distinto, con sistemas de innovación frágiles, lo que implica (Dayton-Johnson, 2009); (RICYT/OEA/CYTED, 200I): I) bajo grado de interacción de empresas (pequeñas y grandes) con el sistema de innovación. 2) poco contacto con instituciones tecnológicas y científicas, 3) menos inversión en I+D y más innovación organizacional y de comercialización de nuevos productos.

En esta región, en muchas empresas la innovación básicamente se trata de cambios incrementales con poco o ningún impacto en los mercados internacionales, basados principalmente en transferencia de tecnología e imitación como el caso de adquisición de maquinaria y equipo y compra de tecnología no incorporada. Cuando se da la transferencia de tecnología extranjera, las empresas que la reciben solamente pueden utilizarla si hacen una inversión en adaptación y aprendizaje (capacidad de absorción), de no hacerlo y de no generar tecnología propia, entonces el resultado es nulo crecimiento, estancamiento, rezago y aislamiento porque ni siquiera se implementa tecnología extranjera (Borondo, 2008). Entonces, podemos decir que la región se encuentra en una etapa de "transición", donde la coyuntura se encuentra en aumentar el ritmo de crecimiento de innovación vertical y horizontal con nuestras propias empresas o a través de Inversión Extranjera Directa (IED) que traiga al país la tecnología y el conocimiento. Esto nos mantendría como países "intermedios" en donde las instituciones estimulan la imitación o implementación de innovaciones y tecnologías extranjeras, propiciando la posibilidad de disminuir las desigualdades comunes cuando la distancia con la frontera del conocimiento es muy amplia. Sin embargo, la fase de transición no puede considerarse un fin, porque en la medida que esta etapa se desarrolla, entonces la innovación se vuelve fundamental para sobrevivir (Borondo, 2008).

\section{El proceso de una convergencia eficaz hacia la inno- vación industrial}

Los países que invierten más en I + D, acceden a la frontera del conocimiento, con la posibilidad de dar un salto tecnológico, el crecimiento en los países más atrasados tiende a ser mayor que en los más avanzados, obteniéndose como resultado la convergencia (Borondo, 2008). Las empresas que invierten en conocimiento son las que innovan y son más productivas (Crespi y Zuniga, 20I2). Esto significa que existe una urgente necesidad de políticas más eficaces para estimular la inversión en innovación, como un camino para empezar a cerrar la brecha que separa a las economías latinoamericanas.

Lo que se debe considerar en la ecuación, es que los determinantes de la innovación no son iguales en todos los países, por lo que se requieren estrategias personalizadas para cada uno de ellos. Además, es necesario considerar aspectos de protección internacional de la propiedad intelectual, las formas de transferencia de conocimiento y su efecto en el crecimiento de nuestros países; a un nivel macro el diseño de incentivos sobre la innovación e incluso los incentivos a la investigación en centros y universidades y a un nivel micro, lo que Katragadda (2006) denomina como prácticas de innovación individual.

Por lo anterior, es necesario considerar varios aspectos clave en el proceso de convergencia hacia una innovación industrial eficaz. Estas actividades dependen principalmente de su capacidad de crear valor único y diferenciado, a partir de los vínculos que se tienen con fuentes de información y conocimientos, tecnologías, prácticas, recursos humanos y financieros. Además de la capacidad de la organización para asimilarlos y ponerlos en práctica a través del diseño e ingeniería industrial, tecnología, patentes, licencias de innovaciones, así como formación o mejora del proceso de comercialización (OECD \& Eurostat, 2005).

A continuación se analizan cinco aspectos que hemos considerado preponderantes en el desarrollo de la innovación industrial de la región: I) Desarrollo de la capacidad de integrar conocimiento multidisciplinario en la innovación, 2) la necesidad urgente de sumarse a un enfoque innovador sustentable y sostenible, 3) la articulación de los dominios macro, meso y micro, 4) el incremento de las prácticas de innovación organizacional e individual y 5) El desarrollo de redes de innovación. Sin embargo cabe mencionar que pueden incluirse otros igualmente valiosos, por lo estos aspectos no son limitativos ni exhaustivos. 


\section{Diseño \\ Desarrollo de la Capacidad de integrar conocimien- to multidisciplinario}

Implica una mayor apertura del uso de metodologías de ingeniería a los problemas socio-industriales, como se ha hecho en la gestión de la cadena de suministro y la operación de procesos complejos. El objetivo es que a través de la innovación se genere una mejor creación de valor frente a un entorno de riesgo e incertidumbre, a través de la atención de nuevos mercados, nuevos requerimientos por parte de los clientes, nuevas tecnologías y nuevos impactos de negocio. Funabashi y Homma (2009) sugieren seis estratos de interacción que permitirían sistematizar la innovación industrial: individuos, operaciones del negocio, gobierno corporativo, creación de valor, ecología industrial y sociedad.

La convergencia de una industria implica la innovación estratégica para el desarrollo sostenible de la ventaja competitiva, por ello ésta es un paradigma del cambio global e implica el desarrollo de un campo y aprender a aplicarlo en otro. La convergencia puede enriquecer a toda la cadena de valor evolucionando en seis niveles (Lee, Olson y Trimi, 20l0): I) del componente, producto o servicio, 2) funcional, 3) organizacional, 4) de tecnología, 5) de la industria y 6) de sistemas biológicos $y$ artificiales.

\section{Enfoque en la Innovación sustentable/sostenible}

La invención y la innovación han servido como un medio para mejorar la calidad de vida de la personas, aunque con mayor competencia, se han acortado los ciclos de vida de los productos, haciendo frágil la ventaja competitiva. Esto hace necesaria la innovación continua para mantener nuevas creaciones de valor y con ello expandir la economía, incrementar el PIB y la riqueza. Sin embargo, con ello también han aparecido consecuencias no deseadas. Enfoques recientes consideran que el crecimiento debe ser entendido como una forma de valorar más equilibradamente los derechos económicos, ambientales y la calidad social (Van den Berg, 2010).

El desarrollo sostenible de las sociedades humanas es altamente complejo, que traducido en tecnologías "verdes" no suele ser inmediatamente rentable y requiere de convergencia y redes que lleven a sinergias entre proveedores y mercados (Pérez, 20I2). Tan relevante es el tópico que ya debe estar insertado en la agenda de preparación de ingenieros. Vest (2005) sostiene que si ha sido importante la fabricación, diseño y desarrollo de productos, pero ahora también lo es la energía, el medio ambiente y la sostenibilidad. Los países latinoamericanos deben apreciar y aprovechar la riqueza de la diversidad con la que cuentan, ampliar los enfoques de ética y responsabilidad social en los sistemas organizacionales, ser expertos en desarrollo y fabricación de productos de alta calidad sin llegar a generar un fenómeno de "agotamiento por innovación", que consiste en realizar grandes esfuerzos orientados a la innovación, pero perdiendo en el camino la claridad y el convencimiento de los objetivos a diferentes plazos y cayendo en el “yo también” (Greenstein, 2013).

Muchas veces, son las condiciones adversas las que llevan a los países, sus organizaciones y ciudadanos a desarrollarse. Senor y Singer (2009) describen como Israel, un país mayormente desértico, en constante estado de guerra y con unos 7.I millones de habitantes tiene la mayor cantidad de Start-Ups (tecnología de nueva generación) e industria de capital de riesgo per cápita en el mundo. Los Start-Ups son compañías fundadas con un claro espíritu emprendedor asociadas a la innovación y al desarrollo de nuevas tecnologías.

Existe una preocupación científica por la convergencia de la transición hacia la sostenibilidad, pero esto requiere cambios radicales y sistémicos en valores, creencias, comportamientos sociales, niveles de gobierno y sistemas de gestión. Esta necesidad involucra potenciar la creatividad humana y el potencial de innovación. En la historia reciente, la invención (creación e implementación de nuevas ideas) y la innovación (propagación en la sociedad) han ido juntas (Westley, et al., 20I I).

La innovación es impulsada por la demanda, por una sociedad que valora la continuidad, lo nuevo y experimentar la transición de lo desconocido a lo conocido (Lane, Pumain y van der Leeuw, 2009), por lo que se estimula la demanda de los nuevos productos y se crean necesidades $y$, cuando surgen problemas sociales o ecológicos radicales, nuestra capacidad de respuesta para innovar se ve obstaculizada por nuestra dependencia de la trayectoria tecnológica y la cultura de innovación vinculada a diversos ámbitos (social, legal, económico, etc.) (Westley, et al., 20II).

Cada vez se evidencia más la necesidad del desarrollo de otro tipo de estrategias que generen mayor valor social aunado a su valor económico, bajo la presión de actores como las ONGs bajo sus intereses sociales y los propios stakeholders con intereses económicos. La realidad es que las normas actuales que rigen el sector privado y la economía no son aptas para apoyar la innovación para la sostenibilidad. Nuestra cultura de consumo tampoco contribuye al desarrollo de sistemas sostenibles (Westley, et al., 20 I I). En una época de recorte de presupuestos y escasez de recursos, se busca incrementar la eficiencia de los sistemas y muchos están tratando de hacerlo a través de la tecnología y la innovación en sus ámbitos, lo que debemos cuidar es no perder en el proceso lo verdaderamente importante: las razones por las que se adopta la innovación, qué valor agregado genera y hacia dónde nos puede conducir como sociedad (Greenstein, 20l3). 


\section{Procedimiento}

\section{Articulación en diferentes dominios: Macro- Meso- Micro}

La dinámica innovadora interactúa con sistemas complejos dentro de la industria global, regional y nacional turbulenta, aunque tienden a existir megatendencias, que permean directa $\circ$ indirectamente a las organizaciones (Arvis, et al., 20I2).

A nivel macro se requiere el cambio de nuestras instituciones globales y nacionales hacia patrones sostenibles y resilientes. Mucho se ha discutido sobre la resilencia desde distintas disciplinas, sin embargo el elemento común suele ser que es una capacidad de recuperación o de respuesta a lo inesperado, a los cambios imprevistos o a los disturbios, generando la capacidad para adaptarse y responder a estos cambios, lo que contribuye a la restauración de la estabilidad de los sistemas complejos (Ozgur, et al., 20I2). Tener un gobierno ineficaz, inestabilidad nacional, un clima pobre de negocios y deficiencias estructurales frenan las posibilidades de inversión sin embargo, la falta de las capacidades necesarias en términos de competencias y esfuerzo por lograr un desarrollo tecnológico parecen ser claves para obstaculizar la inserción en redes de colaboración global.

A un nivel meso o del dominio del problema, se necesitan crear oportunidades para incorporar la innovación y, a una microescala de nivel individual y grupos pequeños, se deben fomentar mecanismos y agencia que pueda conectar la provisión de invención saludable, con las oportunidades institucionales que emergen (Westley, et al., 20I I). En este sentido, desde el dominio de los negocios, la tecnología y el comportamiento organizacional se ha establecido la aproximación de la innovación de arriba hacia abajo, pero la innovación ocurre en las líneas frontales, a nivel de piso y en grupos pequeños, de arriba se proveen los recursos y las oportunidades para explorar y experimentar (Westley, et al., 20I I).

Mirando hacia el 2025 y estableciendo metas para obtener como países y región un mejor "pedazo del pastel", uno de los mayores desafíos es competir mejor en los mercados mundiales, para ello se necesita identificar y predecir mejor las tendencias. Chesbrough (2003) de Princeton encontró hace años que la constante que generalmente subestimamos es el ritmo de los cambios tecnológicos y sobre-estimamos la tasa de cambio social. Esto implica que el impulso del cambio tecnológico, no puede olvidarse de que el trabajo se da en un contexto social, económico, político y de desarrollo complejo. Comparando con el pasado, éste es probablemente el periodo más emocionante en la historia de la humanidad para el desarrollo de la ciencia y la ingeniería (Vest, 2005). En esta era del conocimiento, Latinoamérica no puede seguir dependiendo de su localización geográfica con los mercados, de sus recursos naturales o de la mano de obra barata para crecer. Su mayor desafío se encuentra en el desarrollo de las capacidades intelectuales y organizacionales, el acceso a fondos públicos y privados, así como de la innovación en múltiples áreas de aplicación.

\section{Incremento de las prácticas de innovación organiza- cional e individual en la investigación industrial}

En una era donde hemos vivido la revolución de la información, infraestructuras soportadas en las tecnologías de información y comunicación (TICs) nos han legado herramientas avanzadas $y$ accesibles que abren la posibilidad de ser aprovechadas hacia la interconexión interior y exterior de la organización, estableciendo redes de colaboración y facilitando el desarrollo y la prueba de procesos y productos con cierto grado de innovación por parte de organizaciones de mediano o pequeño tamaño y de personas (Pérez, 20I2). El mundo ha visto el impulso y crecimiento de emprendimientos asiáticos a través de distintos modelos de negocio que han tenido efectos en reducción de costos, empleo masivo e incremento en el volumen de producción. No obstante, más allá de las repercusiones de alto interés económico y de ingeniería industrial, un aspecto relevante para desarrollar liderazgo en innovación es de índole cultural,Vest (2005) lo describe en términos de que algunos países asiáticos tienen tanta hambre de innovación, no tienen vergüenza de aprender todo lo mejor que el mundo tiene para ofrecer y luego tratan de mejorarlo, entendiendo que hay una gran competencia global por lo que no hay tiempo para la complacencia. Con la globalización, paulatinamente también sobrevino un proceso de aprendizaje. Quienes integran empresas que han tenido este tipo de experiencias, particularmente de Asia han desarrollado conocimiento intensivo como proveedores de cadenas de suministros globales e incluso unas, especialmente en dicha región, se han convertido en corporaciones líderes mundiales en sus respectivas industrias, adquiriendo empresas y siendo ellas quienes subcontratan tanto en países avanzados como en desarrollo (Arvis, et al., 20I2); (Pérez, 20I2).

Katragadda (2006) enfatiza que a nivel organizacional un elemento crítico de inicio es contar con una estrategia de innovación donde se involucren a los stakeholders, las unidades de negocio, la alta dirección y los miembros de la organización y donde se establezcan objetivos y medidas de desempeño claras, apoyados de herramientas como el balance scorecard u otras. Sin embargo, a nivel individual se requiere de ciertas actitudes y acciones, en esencia de: libertad para crear (como un niño), cultivarse o educarse con pasión (como un maniaco) y trascender con objetividad (como un guerrero). En 2005, Charles Vest incentivaba a los ingenieros estadounidenses a hacer dos cosas: I) Descubrir nuevos conocimientos científicos y aprovechar el potencial 
tecnológico a través de la investigación y, 2) Desarrollar una tecnología sofisticada más rápidamente y mejor que nadie. Vest (2005) describe como una obligación hacer nuevos descubrimientos, innovar continuamente bajo el principio de la escalabilidad y a apoyar el desarrollo de industrias más sofisticadas (bio/nano/info) y sistematizadas a fin de integrar sistemas e ingeniería, lo que demanda entender mejor los problemas más importantes que enfrentan la región y el mundo, y con ello realizar trabajos más interdisciplinarios, resultando en invenciones, tecnologías, servicios e incluso convergencias de diferentes complementos o aplicaciones de esfuerzos intra-emprendedores (intrapreneurship) o de colaboraciones interinstitucionales (Arvis, et al., 20I2).

\section{Desarrollo de Redes de innovación}

Las organizaciones saben que la innovación cerrada puede ser muy lenta y costosa, por lo que en las últimas décadas ha sido común desarrollar proyectos de innovación abierta en los ecosistemas de negocios (Chesbrough, 2003). Este tipo de paradigma requiere para su funcionamiento soportarse en una plataforma de redes internas, externas y colaborativas interdependientes que generan valor organizacional y compartido por lo que también se le ha denominado Coinnovación (Arvis, et al., 2012).

Por ejemplo, los exportadores dinámicos buscan integrarse a las redes globales de producción desarrollando ciertas competencias básicas y capacidades locales que agreguen valor al proceso innovador sea a través de la capacitación de sus ingenieros, las instituciones científicas, el aprovechamiento de políticas gubernamentales agresivas en materia de I+D, la reputación de las redes en las que se ha insertado, los clusters de conocimiento, etc. En este sentido, la IED puede desempeñar un papel fundamental en el proceso de conversión, pero se requieren las condiciones y políticas adecuadas. También, algunos distritos industriales funcionan con un conjunto de estrategias de subcontratación, outsourcing, redes horizontales y offshoring, entre otros para que empresas altamente especializadas produzcan de manera flexible y eficiente, sabiendo que vía el modelo de cluster, juntos pueden enfrentar la volatilidad de los mercados, los picos de producción y satisfacer las necesidades cambiantes de la demanda (Capasso y Morrison, 2013). Este tipo de distritos han demostrado ser un medio dinámico para las actividades de innovación facilitando la cooperación, la especialización y la explotación del conocimiento formal e informalmente (Maskell, 200I).

La conversión a la co-innovación inicia con ideas y un acuerdo de colaboración que requiere del compromiso, la experiencia y la co-creación de valor, aspectos difíciles de ser imitados por los competidores. En este sentido, es posible innovar en (Arvis, et al., 2013): I) la introducción de nuevos productos o servicios o la convergencia de complementos o aplicaciones, 2) la eficiencia de la arquitectura de la cadena de valor, mejorando la calidad de procesos y productos vía just-in-time, TQM, Six Sigma, Lean Manufacturing, etc., 3) la reinvención de valor al cliente, donde la co-creación se gesta con los clientes a fin de crear valor compartido, 4) la creación de valor para expandir la base de clientes vía las comunidades de clientes y otras herramientas tecnológicas que usan los clientes. Este tipo de innovación tiene como propósito crear valor diferenciado para grupos específicos de clientes y por último, 5) creación de valor a través de nuevos modelos de negocio.

\section{Conclusiones}

En gran medida, la innovación en Latinoamérica considera principalmente el uso de la capacidad de absorción y la adaptación de otras creaciones tecnológicas. Sin embargo, la intensa competencia del mundo globalizado somete a una mayor presión a las organizaciones industriales a cambiar su dinámica, al desarrollar nuevos productos y procesos, utilizar nuevas tecnologías y encontrar nuevos mecanismos para responder a cadenas de valor más integradas y coresponsables de la generación de competitividad industrial. Sin embargo, la región aún tiene enormes desafíos que atender no solamente para mantener, sino para mejorar su posición en la siguiente década. La apertura política, económica, tecnológica y social no serán suficientes para lograr la competitividad, en general, la región requiere trabajar en el fomento de una cultura de innovación, así como una reinvención de su estrategia industrial bajo el esfuerzo de cambio de distintos actores, el rol de la política gubernamental, la inversión en infraestructura, las alianzas e inversión extranjera directa (IED) de organizaciones de mayor envergadura tecnológica, el desarrollo de nuevas habilidades y rutinas dentro de la organización industrial, la reconfiguración del currículo en la formación de ingenieros en las universidades son algunos de los aspectos clave en la construcción de un sistema formal de innovación y aprendizaje donde se absorbe y comparte el conocimiento y luego se mejora con el tiempo.

La innovación es un imperativo para desarrollar competitividad y tener éxito en los mercados (Arvis, et al., 2012). El país que no se inserte estratégicamente visionando más allá de la política industrial y tecnológica, comprendiendo que esta tarea requiere un compromiso de largo plazo, que implica inversión y costos, y proyectando que llevará tiempo obtener resultados observables en bienestar de los ciudadanos, está condenado a la desarticulación y erosión del bienestar de los pobladores y los recursos de su nación. 


\section{Referencias}

ÁlVAREZ, R., Bravo, C., Navarro, L. (2010). Innovation and productivity in Chile. IDB working paper no. 190. Washington, DC, United States: Inter-American Development Bank. http://idbdocs.iadb.org/wsdocs/getdocument. aspx?docnum $=35423592$ [Accessed November 20, 2013]

ARVIS, J. F., Mustra, M.A., Ojala, L., Shepherd, B., Saslavsky, D. (20I2). Connecting to Compete 20I4. Trade Logistics in the Global Economy. The Logistics Performance Index and Its Indicators. Washington: The International Bank for Reconstruction and Development/The World Bank.

ARZA, V., López, A. (2010). Innovation and productivity in the argentine manufacturing sector. IDB working paper no. 187. Washington, DC, United States: Inter-American Development Bank. http://www.iadb.org/res/publications/pubfiles/ pubIDB-WP-I87.pdf [Accessed September 15, 20I3]

BELL, M., Pavitt, P. (1993). Technological accumulation and industrial growth: Contrasts between developed and developing countries. Industrial and Corporate Change, 2(2), $|57-2| 1$.

BENAVENTE, J. M. (2006). The role of research and innovation in promoting productivity in Chile. Economics of Innovation and New Technology, I54(5), 30 I-3I5.

BORONDO, C. (2008). La innovación en la literatura reciente del crecimiento endógeno. Principios, 12, I I-42.

CAPASSO, M., Morrison, A. (20I3). Innovation in industrial districts: evidence from Italy. Management Decision, $5 \mathrm{I}(6)$, 1225-1249.

CHESBROUGH, H. (2003). Open Innovation: The New Imperative for Creating and Profiting from Technology. Boston, M.A. Harvard Business School Press.

COHEN, W. M., Levinthal, D. A. (1990). Absorptive Capacity: A New Learning Perspective on Learning and Innovation. Administrative Science Quarterly, 35 I28-I52.

COLLINS, J., Hitt, M. (2006). Leveraging tacit knowledge in alliances: The importance of using relational capabilities to build and leverage relational capital. Journal of Engineering and Technology Management Jet-M, 23, I47-167.

CRESPI, G., Zuniga, P. (20I2). Innovation and Productivity: Evidence from Six Latin American Countries. World Development, 40(2), 273-290.
DAYTON-JOHNSON, J. (2009). Innovación en América Latina: Una visión desde la perspectiva de la OCDE. LATAM Innovation Index Project.

DYNAMICS (39), 3, 2I8-225. (20I2). Co-innovation: convergenomics, collaboration, and co-creation for organizational values, Management Decision, 50(5), 8I7-83I.

FUNABASHI, M., Homma, K. (2009). Transdisciplinary Approach Industrial Innovation Management. ICROS-SICE International Joint Conference 2009, 5I I7-5I 20.

GLOBAL Innovation Index [GII] (20I0). Global Innovation Index 2009-10. INSEAD. http://www.globalinnovationindex. org (20II). Global Innovation Index 20II. INSEAD. http:// www.globalinnovationindex.org (2012). Global Innovation Index 2012. INSEAD. http://www.globalinnovationindex.org (20I3). Global Innovation Index 20 I3. INSEAD. http://www. globalinnovationindex.org [Accessed November 20, 20I3]

GREENSTEIN, D. (20I3). Innovation Exhaustion and a Path to Moving Foward. http://www.insidehighered.com/advice/2013/07/03/essay-how-do-strategic-planning [Accessed November 20, 20I3]

GRUPP, H. (1998). Foundation of the Economics of Innovation:Theory, Measurement, and Practice. UK: Edwards Elgar Publishing, Inc.

KATRAGADDA, G. (2006). Organizational and Individual Innovation Practices in Industrial Research. International Conference on Management of Innovation and Technology (IEEE), 999-1003.

LANE, D., Pumain, D.Van der Leeuw, S. (2009). Complexity perspectives in innovation Leeand social change. New York: Springer.

LEE, S.M., Olson, D.L., Trimi, S. (2010). The impact of convergence on organizational innovation. Organizational Dynamics, 39(3):218-225.

LI, K.;Wang, D. (20I0). Analysis on the Equilibrium of Industrial Innovation Based on Creative Destruction. 3rd International Conference on Information Management, Innovation Management and Industrial Engineering, 207-2 II.

LOGISTIC Performance Index [LPI] (20I4). Logistic Performance Index 2012-14. INSEAD. http://web.worldbank. org/WBSITE/EXTERNAL/TOPICS/TRADE/0,, content MDK:23 I 886 I 3 pagePK:2 $10058 \sim$ piPK:2 $10062 \sim$ theSite PK:23907l,00.html [Accessed March 15, 20l4] 
MASKELL, P. (200I). Towards a knowledge-based theory of the geographical cluster. Industrial and Corporate Change, 10, 919-94I.

OECD (2009). Innovation in firms: A microeconomic perspective. Paris, France: Organization for Economic Cooperation and Development. (20I la). New Doctorate Graduates. En OECD Science, Technology and Industry, Scoreboard 20I I.OECD Publiching. http://dx.doi.org/ $0.1787 /$ sti_scoreboard-20 I I-I2-en (20l Ib). Higher education and basic research. En OECD Science, Technology and Industry Scoreboard 20II. OECD Publishing. http://dx.doi.org//0.1787/ sti_scoreboard-20II-I7-en (20IIc). Mixed modes of innovation. En OECD Science, Technology and Industry Scoreboard 20II. OECD Publishing. http://dx.doi.org//0.1787/ sti_scoreboard-20I I-44-en (20I Id). Broader innovation. En OECD Science, Technology and Industry Scoreboard $201 \mathrm{I}$. OECD Publishing. http://dx.doi.org// 0.1787/sti_scoreboard20II-45-en [Accessed June 08, 20I3]

OECD and Eurostat (2005). The Oslo manual:The measurement of scientific and technological activities. Guidelines for collecting and interpreting innovation data. (3rd ed.), Paris, France: Organization for Economic Cooperation and Development/Eurostat.

OZGUR, Devanandham, Sauser, Mansouri (20I2). Perspectives on Measuring Enterprise Resilience. Systems Conference. 4th Annual IEEE, 587-592.

PÉREZ, C. (2012). Innovation systems and policy: Not only for the rich? Working Papers in Technology Governance and Economic Dynamics, 42, I-19.

RAFFO, J., Lhuillery, S., Miotti, L. (2008). Northern and southern innovativity: A comparison across European and Latin American countries. European Journal of Development Research, 20(2), 219-239.

RICYT/OEA/CYTED (200I). Manual de Bogotá: Normalización de Indicadores de Innovación Tecnológica en América Latina y el Caribe. http://www.uis.unesco.org/Library/Documents/Bogota\%20Manual_Spa.pdf [Accessed June 10,2013]

ROUVINEN, P. (2002). R\&D-productivity dynamics: Causality, lags, and dry holes. Journal of Applied Economics, 5(I), 123-I56.

SENOR, D., Singer, S. (2009). Start-up Nation: The Story of Israel's Economic Miracle. USA:Twelve.

VAN DEN BERG, J. (20I0). Environment versus growth-a criticism of "degrowth" and a plea for "a-growth". Ecological Economics, 70, 88I-890.
VEST, C. M. (2005). Educating Engineers for 2020 and Beyond. http://www.engineeringchallenges.org/cms/7/26/7639. aspx [Accessed May 12, 20I3]

WESTLEY, F., Olsson, P., Folke, C., Homer-Dixon, T., Vredenburg, H., Lookbach, D.,Thompson, J., Nilsson, M., Lambin, E., Sendzimir, J., Barnerjee, B., Galaz,V.,Van der Leeuw, S. (20I I). Tipping Toward Sustainability: Emerging Pathways of Transformation. Ambio, 40,762-780.

WORLD Intellectual Property Organization [WIPO] (2013). PTC Yearly Review. The International Patent System 2013.

ZHAO, Z. J., Anand, J. (2009). A multilevel perspective on knowledge transfer: Evidence from the Chinese automotive industry. Strategic Management Journal, 30 (9), 959-983. 